\title{
Изучение сверхструктуры в сильно легированном пористом фосфиде индия рентгеновскими методами
}

\author{
(C) М.Е. Бойко, М.Д. Шарков, Л.Б. Карлина, А.М. Бойко, С.Г. Конников \\ Физико-технический институт им. А.Ф. Иоффре Российской академии наук, \\ 194021 Санкт-Петербург, Россия \\ E-mail: mischar@mail.ru, boikomix@gmail.com
}

(Получена 23 мая 2017 г. Принята к печати 24 мая 2017 г.)

Образец фосфида индия InP, подвергнутый процедуре порообразования и затем легированный атомами $\mathrm{S}$, исследован методами рентгеновской дифрактометрии и малоуглового рассеяния рентгеновских лучей (МУРР) (при $\mathrm{Cu} K_{\alpha 1}$-излучении). Исходя из данных рентгеновской дифракции, показано, что образец состоит из когерентных (однонаправленных) однородных компонент. С помощью точечного детектора в режиме аномального прохождения по Борману получен ряд кривых малоуглового рассеяния рентгеновских лучей при положениях образца, варьируемых азимутальными поворотами. На основе зарегистрированных данных смоделирована 2D-картина малоуглового рассеяния рентгеновских лучей для исследуемого образца, которая позволила определить трансляционную симметрию кластеров и, следовательно, присутствие сверхструктуры. Определены межплоскостные расстояния в сверхструктуре в направлениях (110) и (110) относительно решетки InP как $\sim 260$ и 450 нм соответственно. Симметрия сверхструктуры определена как $C_{2 v}$ в плоскости решетки образца (001).

DOI: $10.21883 /$ FTP.2018.01.45324.8628

\section{1. Введение}

Свойства полупроводниковых материалов могут существенно изменяться в результате сильного легирования и формирования пор в веществе. Если при этом в образце образовалась сверхструктура (например, сверхрешетка), то ее трансляционная симметрия может привести к появлению дополнительных зон в энергетической структуре материала $[1,2]$. Изучение возможных эффектов, обусловленных комбинацией порообразования и сильного легирования полупроводниковых соединений, может в перспективе улучшить полезные свойства полупроводниковых приборов.

Фосфид индия InP является полупроводниковым соединением с решеткой сфалерита и шириной запрещенной зоны 1.34 эВ. В ФТИ им. А.Ф. Иоффе методом жидкофазной эпитаксии была выращена монокристаллическая пленка InP толщиной 20 мкм с направлением нормали к поверхности (001). Затем пленка была подвергнута процедуре порообразования с одной из сторон, после чего легирована в основном атомами $\mathrm{S}$, с концентрацией примеси около $10^{18} \mathrm{~cm}^{-3}$. Изготовленный таким образом образец был исследован методами рентгеновской дифрактометрии (РД - рентгеноструктурный анализ, РСА) и малоуглового рассеяния рентгеновских лучей (МУРР). Эксперименты проводились на установке МАРС-2 в ФТИ им. А.Ф. Иоффе.

Методы РД и МУРР являются эффективным инструментом изучения фазового состава образца, - в первую очередь размеров и формы однородных фазовых составляющих и их кристаллической решетки $[3,4]$. Важно отметить несколько существенных деталей, связанных с интерпретацией кривых МУРР.

Во-первых, данные МУРР, как правило, представляются в виде зависимости интенсивности рассеяния не от угла, а от волнового вектора $q=4 \pi / \lambda \sin \theta$, где $\lambda$ - длина волны анализирующего пучка рентгеновского излучения, а $\theta-$ угол рассеяния пучка веществом образца $[3,5]$. При построении кривых МУРР, полученных в просвечивающем режиме, $q$ обозначает не модуль волнового вектора, а его проекцию на определенную (направленную) ось, и поэтому кривая может находиться в области как положительных, так и отрицательных значений волнового вектора (так называемое „прохождение через ноль“).

Во-вторых, на кривых МУРР могут присутствовать интенсивные пики, отвечающие структурной периодичности внутри образца с периодами, превосходящими межатомные расстояния на порядки величины (как, например, в слоистых средах и сверхрешетках) [3]. Такие пики обычно называются „брэгговскими“ или „интерференционными“ (кроме отдельного случая, когда они обусловлены практически постоянной толщиной однослойного образца). При этом для положений максимумов подобных пиков вдоль угловой шкалы (либо шкалы волновых векторов), как и для рефлексов РД, выполняется закон Брэгга-Вульфа [3,4].

В-третьих, данные МУРР, полученные в режиме отражения, подвергаются значительной модификации из-за эффекта полного внешнего отражения (ПВО) [6]. Существует такой угол (называемый углом ПВО), что данные МУРР, отвечающие меньшим углам рассеяния, не могут быть проанализированы стандартными способами, описанными в литературе (например, [7]).

\section{2. Эксперимент}

Регистрация данных РД и МУРР проводилась на установке МАРС-2 (ФТИ им. А.Ф. Иоффе) мощностью 6 кВт 
на основе источника рентгеновского излучения Rigaku с вращающимся анодом в соответствии с принципами, описанными в статье [8]. Во всех экспериментах применялось $\mathrm{Cu} K_{\alpha 1}$-излучение с длиной волны пучка $1.54 \AA$. Кривые МУРР измерялись как в режиме отражения, так и в просвечивающем режиме. Все данные были зарегистрированы с помощью точечного детектора сцинтилляционного счетчика, но в просвечивающем режиме при этом была получена серия кривых МУРР, соответствовавших различным вариантам расположения (ориентации) образца в пространстве. В совокупности данные МУРР, зарегистрированные в просвечивающем режиме, образовали единую модель дифракционной 2D картины в области малых углов.

Эксперименты по получению МУРР проводились при такой геометрии, что вектора рассеяния лежали в плоскости (001) и, следовательно, углы падения были близки к нормали поверхности пленки, отвечавшей направлению решетки (001). Поэтому, несмотря на поглощение фотонов пучка веществом образца, благодаря эффекту аномального прохождения (эффекту Бормана) [4] соотношение сигнал/шум у измеренных данных оставалось высоким, на уровне $10^{1}-10^{3}$. В области углов в ближайшей окрестности нулевого значения (направления прямого пучка) требовалось избежать ситуации, когда слишком высокие интенсивности прошедшего пучка могли бы привести к непропорциональным величинам регистрируемого сигнала [9]. В этих целях для понижения интенсивности прошедшего прямого пучка, чтобы детектор работал в области линейной регистрации интенсивности, при измерениях в ближайшей окрестности рассеянного излучения $\left(|q| \leq 0.01 \AA^{-1}\right)$ перед детектором устанавливался ступенчатый массовый поглотитель.

На рис. 1 проиллюстрирован подход, примененный к измерению данных МУРР в просвечивающем режиме. Кривые дифракционного отражения, у которых

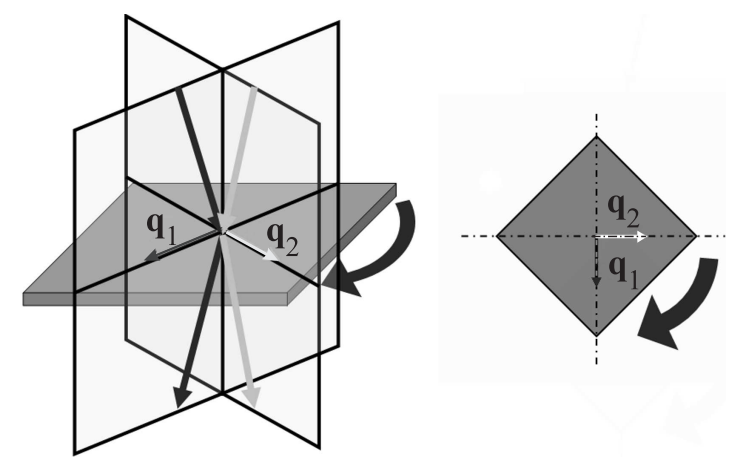

Рис. 1. Общая схема измерения данных МУРР от образца InP в просвечивающем режиме: общий вид (слева), проекция на горизонтальную плоскость (справа). Исходное положение образца позволяет получать данные МУРР при направлении волнового вектора $\mathbf{q}_{1}$. Затем осуществляется поворот образца и регистрация данных МУРР при другом направлении волнового вектора (например, $\mathbf{q}_{2}$ ).

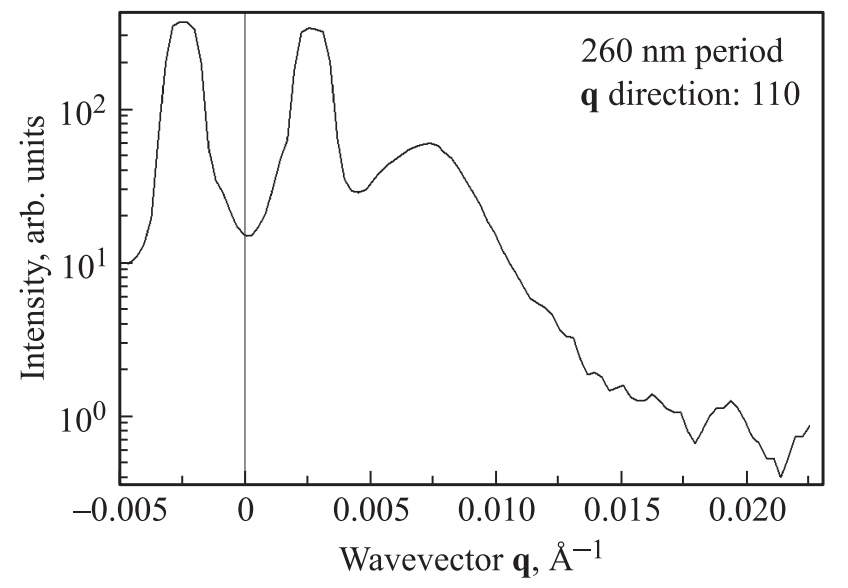

Рис. 2. Кривая МУРP от образца InP, измеренная на $\mathrm{Cu} K_{\alpha 1}$-излучении в просвечивающем режиме при направлении волнового вектора, соответствующего направлению (110) в решетке InP.

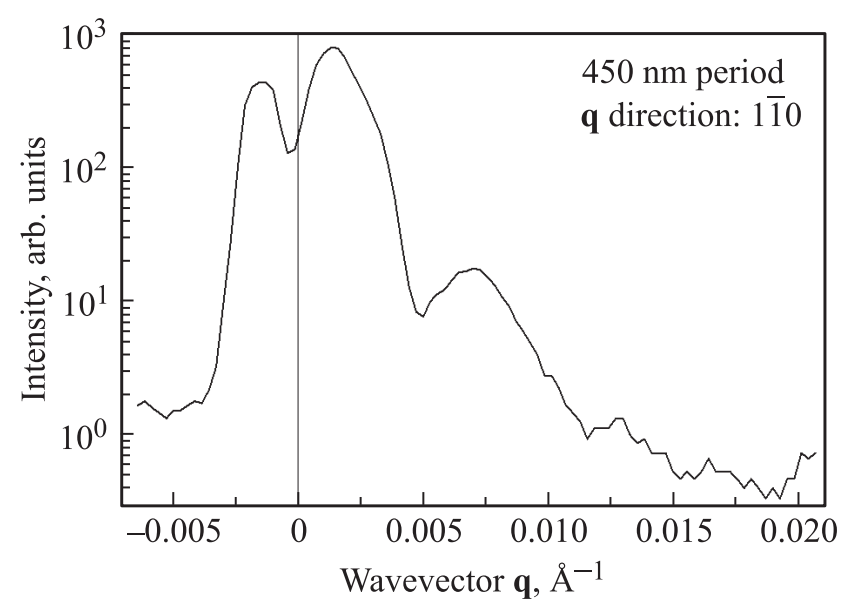

Рис. 3. Кривая МУРP от образца InP, измеренная на $\mathrm{Cu} K_{\alpha 1}$-излучении в просвечивающем режиме при направлении волнового вектора, соответствующего направлению (110) в решетке InP.

вектор рассеяния отвечает взаимно перпендикулярным кристаллографическим направлениям (110) и (1하) в кубической матрице InP, наиболее сильно отличаются от остальных. Эти две кривые показаны на рис. 2 и 3 соответственно.

На рис. 4 в свою очередь приведена кривая МУРР, полученная в режиме отражения со стороны пористой части образца. На рис. 5 показаны данные РД, зарегистрированные также со стороны пористой части образца.

\section{3. Результаты и анализ}

Обе кривые МУРР, показанные на рис. 2 и 3, содержат по крайней мере по одной паре брэгговских пиков, находящихся на противоположных позициях вдоль шкалы волновых векторов относительно нуля. Кривая, приведенная на рис. 2 и соответствующая направлению 
волнового вектора (110), дает при обработке величину периода около $260 \mathrm{нм}$, а вторая кривая (направление (110), рис. 3) приводит к периоду около 450 нм. Смоделированная 2D картина МУРР, основанная на обработке двух вышеописанных кривых (а также на том обстоятельстве, что в иных направлениях волнового вектора интенсивные пики интерференции не наблюдались), показана на рис. 6. Картина подобного вида характерна для сред, которые содержат 2D или 3D сверхструктуру, обладающую в данном случае симметрией группы $C_{2 v}$ (или группы более высокого порядка, с подгруппой $C_{2 v}$ ). Сверхструктура при этом будет иметь плоскую прямоугольную или трехмерную орторомбическую симметрию.

Кривая МУРР, полученная в режиме отражения (рис. 4), содержит широкий пик - характерный признак ПВО. Попытка обработать данную кривую в области углов, превосходящих угол ПВО, на основе модели

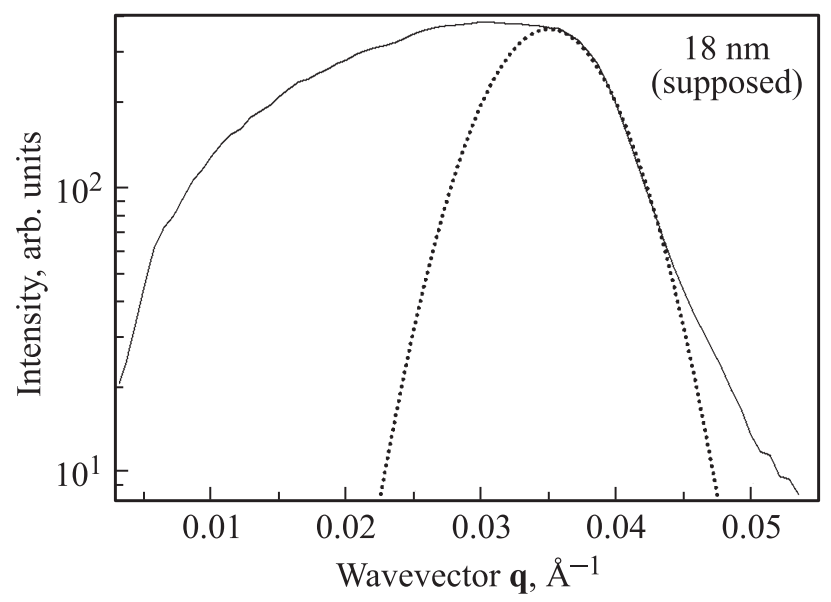

Рис. 4. Кривая МУРР, измеренная на $\mathrm{Cu} K_{\alpha 1}$-излучении в режиме отражения от пористой стороны образца InP. Пунктиром показана модель гипотетического брэгговского пика, частично расположенного в области ПВО.

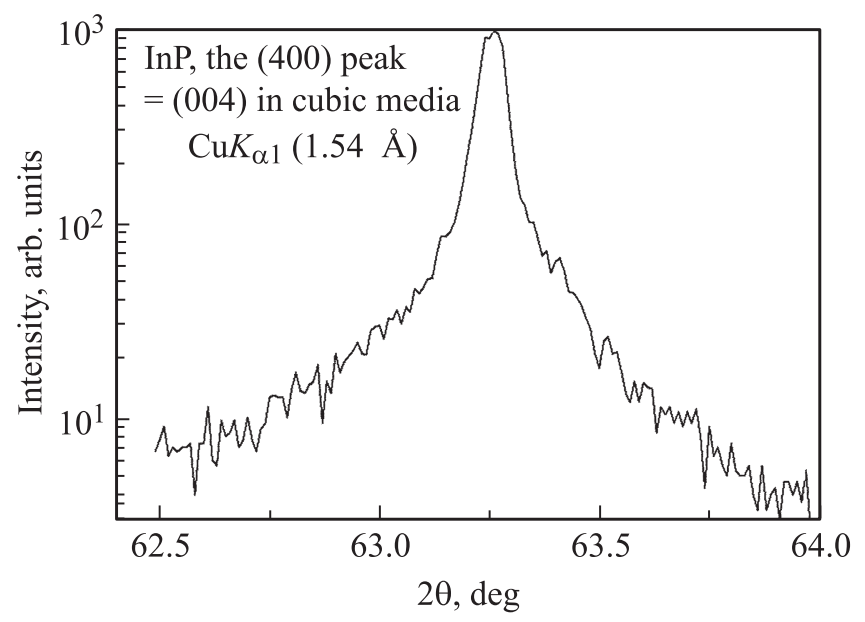

Рис. 5. Данные РД, полученные при $\mathrm{Cu} K_{\alpha 1}$-излучении от пористой стороны образца InP.

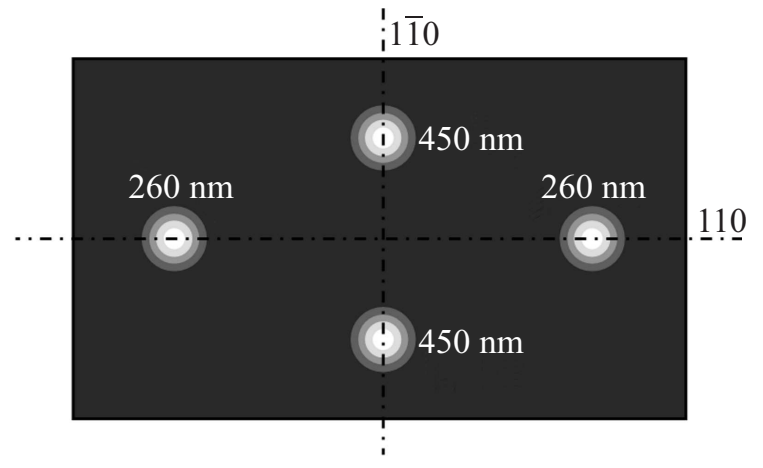

Рис. 6. Модель 2D картины МУРP от образца InP, построенная на основе кривых МУРР, приведенных на рис. 2 и 3.

Порода [7] привела к величине модуля породовского показателя, намного большей типичных значений (от 1 до 4). Таким образом, анализ кривой МУРР по модели Порода не дает адекватной физической интерпретации. Эта ситуация может быть объяснена возможным присутствием на кривой брэгговского (интерференционного) пика, частично расположенного в диапазоне углов ниже угла ПВО. Модель, позволяющая получить оценку формы брэгговского пика по фрагментам кривой МУРР, находящимся выше угла ПВО, дала положение максимума пика, отвечающее расстоянию около 18 нм.

На рис. 5 показан фрагмент данных РД, в котором присутствует дифракционный пик, соответствующий рефлексу (400), а также симметричному отражению (004) в кубической атомной матрице InP. Пики, отвечающие другим направлениям вектора рассеяния, на полной дифрактограмме отсутствуют. Отсюда следует, что образец либо остался монокристаллическим после процессов порообразования и легирования, либо состоит из одинаково ориентированных в пространстве фазовых компонент (так называемых когерентных фаз).

\section{4. Заключение}

Образец после проведения над ним процедур порообразования и легирования атомами $\mathrm{S}$ представляет собой либо монокристалл InP, в котором нормаль к пленке ориентирована вдоль направления (001), либо состоит из одинаково ориентированных (когерентных) зерен InP.

Распределение неоднородностей (примесных фаз или пор) внутри образца соответствует кристаллическому (периодическому) типу. Присутствует сверхструктура, обладающая симметрией группы $C_{2 v}$ в плоскости (001). Межплоскостные расстояния сверхструктуры составляют 260 нм вдоль направления (110) в исходной матрице фосфида и 450 нм в направлении (1ㅣㅜ).

В направлении (001) относительно исходной матрицы InP в образце может существовать слоистость (периодичность) с величиной периода около 18 нм. 


\section{Список литературы}

[1] Л.В. Келдыш. ФТТ, 4 (8), 2265 (1962).

[2] L. Esaki, R. Tsu. IBM J. Res. Develop., 14, 61 (1970).

[3] М.Е. Бойко, М.Д. Шарков, А.М. Бойко, С.Г. Конников, А.В. Бобыль, Н.С. Будкина. ЖТФ, 85 (11), 1 (2015).

[4] В.И. Иверонова, Г.П. Ревкевич. Теория рассеяния рентгеновских лучей (М., изд. МГУ, 1978).

[5] Д.И. Свергун, Л.А. Фейгин. Рентгеновское и нейтронное малоугловое рассеяние (М., Наука, 1986).

[6] Л.Д. Ландау, Е.М. Лифшиц. Электродинамика сплошных сред (М., Физматлит, 2005) с. 390.

[7] O. Glatter, O. Kratky (eds). Small-Angle X-Ray Scattering (London, Academic, 1982).

[8] M.D. Sharkov, K.Ju. Pogrebitsky, M.E. Boiko, S.G. Konnikov. Precision Instrument and Mechanology, 1 (2), 32 (2012).

[9] H. Schnablegger, Y. Singh. The SAXS Guide: Getting Acquainted with the Principles (Graz, Anton Paar GmbH, 2013).

Редактор Г.А. Оганесян

\section{Exploring superstructure in high doped porous indium phosphide via $X$-ray techniques}

M.E. Boiko, M.D. Sharkov, L.B. Karlina, A.M. Boiko, S.G. Konnikov

loffe Institute, 194021 St. Petersburg, Russia

Abstract A sample of indium phosphide InP subjected to the pore generation procedure and then doped by $\mathrm{S}$ atoms was studied via the techniques of $X$-ray diffractometry (XRD) and smallangle $X$-ray scattering (SAXS). XRD data showed the sample to consist of aligned homogeneous components. In such a mode of transmission SAXS measuring when the analyzed beam was weakly attenuated due to the Borrmann effect, a set of SAXS curves in different sample positions consequently changed by sample azimuth rotations were registered with the help of a point detector. Basing on the obtained SAXS data sets the explored sample 2D SAXS pattern which allowed one to define the longdistance translation symmetry and, conclusively, the superstructure presence within the sample was simulated. The superstructure interplanar distances in the InP matrix directions (110) and (11 0$)$ were determined as about $260 \mathrm{~nm}$ and $450 \mathrm{~nm}$, respectively. The superstructure symmetry group was found to be $C_{2 v}$ versus the (001) sample lattice plane parallel to the sample surface. 